\title{
Analytical solution to the nonlinear singular boundary value problem arising in biology
}

Abdollah Dinmohammadi ${ }^{1}$, Abdolrahman Razani ${ }^{2}$ and Elyas Shivanian ${ }^{2 *}$

"Correspondence:

eshivanian@gmail.com

${ }^{2}$ Department of Mathematics, Imam

Khomeini International University,

Qazvin, 34149-16818, Iran

Full list of author information is

available at the end of the article

\begin{abstract}
This paper proposes an analytical procedure for the nonlinear singular boundary value problem that arises in biology and in the study of some diseases. As a first step, we present a constructive proof of the existence and uniqueness of solution. Then, we apply the Picard iterative sequence by constructing an integral equation whose Green's function is not negative. The convergence of this iterative sequence is then controlled by an embedded parameter so that it tends to the unique solution.
\end{abstract}

Keywords: singular boundary value problem; Picard iterative sequence; biology

\section{Introduction}

Consider the two-point boundary value problem of the type

$$
\begin{aligned}
& y^{\prime \prime}(x)+n y^{\prime}(x)+\frac{m}{x} y^{\prime}(x)=f(x, y(x)), \quad 0<x \leq 1, m>0, n \in \mathbb{R}, \\
& y^{\prime}(0)=0, \quad A y(1)+B y^{\prime}(1)=C,
\end{aligned}
$$

which arises in biology and physiology problems. In boundary conditions (1.2), we have, in fact, $A \neq 0, A$ and $B$ have the same sign and $C \in \mathbb{R}$, but it is supposed, without loss of generality, $A>0$ and $B, C \geq 0$ (if $C<0$, then we can apply $y \rightarrow-y$ ). It is supposed that $f(x, y(x))$ is non-singular with respect to the independent variable $x \in[0,1]$ but it can be singular with respect to $y$.

The general form of the nonlinear diffusion problem is given by [1]

$$
\begin{aligned}
& \frac{1}{p(x)}\left(p(x) g(x, y) y^{\prime}\right)^{\prime}=F(x, y), \quad 0<x \leq 1, \\
& y^{\prime}(0)=0, \\
& y(1)=h\left(y(0), y(1), y^{\prime}(1)\right) .
\end{aligned}
$$

If it is supposed

$$
g(x, y)=\exp (n x), \quad p(x)=x^{m}, \quad F(x, y)=\exp (n x) f(x, y)
$$

(c) The Author(s) 2017. This article is distributed under the terms of the Creative Commons Attribution 4.0 International License (http://creativecommons.org/licenses/by/4.0/), which permits unrestricted use, distribution, and reproduction in any medium, provided you give appropriate credit to the original author(s) and the source, provide a link to the Creative Commons license, and indicate if changes were made. 


$$
h\left(y(0), y(1), y^{\prime}(1)\right)=-\frac{B}{A} y^{\prime}(1)+\frac{C}{A},
$$

then boundary value problem (1.1)-(1.2) will be attained. Boundary value problem (1.1)(1.2), in some special cases, with $m=0,1,2$ and $n=0$ has been studied in [2-11].

On the other hand, boundary value problem of the form (1.1)-(1.2) involving the governing ordinary differential equation (1.1) or slight generalizations of it has been investigated by Gatica et al. [12], Fink et al. [13], Baxley [14], Baxley and Gersdorff [15], Wang and Li [16], Tinio [17], Wang [18], Ebaid [19], Agarwal and O'Regan [20] and also by the authors of $[21,22]$. In these works, the fixed point theory or approximation theory was prevalently used, and their studies included assumptions that restrict $f(x, y)$ to be of one sign and usually continuous for $y \geq 0$. Later, in Refs [23-25], authors started to allow sign-changing nonlinearities but still they have required $f(x, y)$ to be continuous for $y \geq 0$. We notice that, in all these works, it has been assumed $n=0$ and they have considered some particular types of boundary conditions (1.2) or some specific forms of governing differential equation (1.1).

The aim of the present work is to provide some constructive existence and uniqueness theorems for problem (1.1)-(1.2) of the same type as those provided by Ford and Pennline in [26] by the same assumptions considered by them, i.e., $f(x, y(x))$ is not only allowed to be sign-changing but it also can be singular with respect to $y$. Also, we apply the Picard iterative sequence by constructing an integral equation whose Green's function is not negative. The convergence of this iterative sequence is then controlled by an embedded parameter so that it converges to the unique solution.

\section{Constructing the integral equation}

We subtract $k^{2} y$ from both sides of equation (1.1) so that the differential equation is converted to

$$
y^{\prime \prime}(x)+n y^{\prime}(x)+\frac{m}{x} y^{\prime}(x)-k^{2} y(x)=f(x, y(x))-k^{2} y(x) .
$$

Let us now consider the homogeneous type of the above equation, i.e.,

$$
\omega^{\prime \prime}(x)+n \omega^{\prime}(x)+\frac{m}{x} \omega^{\prime}(x)-k^{2} \omega(x)=0, \quad m>0, n \in \mathbb{R},
$$

with two homogeneous conditions

$$
\omega^{\prime}(0)=0, \quad A \omega(1)+B \omega^{\prime}(1)=0 .
$$

Suppose that $u_{m}(x)$ and $v_{m}(x)$ are given as follows:

$$
\begin{aligned}
& u_{m}(x)=e^{\frac{1}{2} x\left(-\sqrt{4 k^{2}+n^{2}}-n\right)} L_{\alpha}^{m-1}\left(x \sqrt{4 k^{2}+n^{2}}\right), \quad \alpha=-\frac{m\left(\sqrt{4 k^{2}+n^{2}}+n\right)}{2 \sqrt{4 k^{2}+n^{2}}}, \\
& v_{m}(x)=e^{\frac{1}{2} x\left(-\sqrt{4 k^{2}+n^{2}}-n\right)} U\left(\beta, m, \sqrt{4 k^{2}+n^{2}} x\right), \quad \beta=\frac{m\left(n+\sqrt{4 k^{2}+n^{2}}\right)}{2 \sqrt{4 k^{2}+n^{2}}},
\end{aligned}
$$

where $L_{\alpha}^{m-1}$ is the generalized Laguerre polynomial which is related to hydrogen atom wave functions in quantum mechanics. Further, $U(a, b, z)$ is the hypergeometric func- 
tion which is a second linearly independent solution to Kummer's equation and is defined by

$$
U(a, b, z)=\frac{1}{\Gamma(a)} \int_{0}^{\infty} t^{a-1}(t+1)^{b-a-1} e^{-t z} \mathrm{~d} t
$$

Now, we define

$$
\begin{aligned}
& u(x)=u_{m}(x), \\
& v(x)=v_{m}(x)-S u_{m}(x), \quad S=\frac{A v_{m}(1)+B v_{m}^{\prime}(1)}{A u_{m}(1)+B u_{m}^{\prime}(1)} .
\end{aligned}
$$

It can be easily seen that $u(x)$ and $v(x)$ are the solutions of Eq. (2.2) so that $u^{\prime}(0)=u_{m}^{\prime}(0)=0$ and $A v(1)+B v^{\prime}(1)=0$ hold, respectively. We will give some properties of these functions in the next section.

Assume that $\varphi(x)$ satisfies

$$
\begin{aligned}
& \varphi^{\prime \prime}(x)+n \varphi^{\prime}(x)+\frac{m}{x} \varphi^{\prime}(x)-k^{2} \varphi(x)=0, \\
& \varphi^{\prime}(0)=0, \quad A \varphi(1)+B \varphi^{\prime}(1)=C .
\end{aligned}
$$

In fact, $\varphi(x)$ can be expressed in terms of $u(x)$ as follows:

$$
\varphi(x)=\frac{C u(x)}{A u(1)+B u^{\prime}(1)}
$$

Now, consider differential equation (2.1) with the homogeneous boundary conditions

$$
y^{\prime}(0)=0, \quad A y(1)+B y^{\prime}(1)=0 .
$$

Then, for $k^{2} \neq 0$, Eq. (2.1) can be converted to an equivalent integral equation by means of the Green's function appropriate to the operator on the left-hand side as follows:

$$
y(x)=\varphi(x)+\int_{0}^{1} G(x, t)\left[k^{2} y(t)-f(t, y(t))\right] \mathrm{d} t,
$$

where $G(x, t)$ satisfies

$$
\begin{aligned}
& G_{x x}+n G_{x}+\frac{m}{x} G_{x}-k^{2} G=-\delta(x-t), \\
& G_{x}(0, t)=0, \quad A G(1, t)+B G_{x}(1, t)=0,
\end{aligned}
$$

where $\delta(x)$ is the Dirac delta function. It can be easily discovered from the elementary theory of differential equations that $G(x, t)$ may be expressed as

$$
G(x, t)=\frac{1}{W(t)} \begin{cases}u(x) v(t), & x \leq t \\ v(x) u(t), & x \geq t\end{cases}
$$


where $u(x)$ and $v(x)$ are given by Eqs. (2.7)-(2.8) and the Wronskian $W(t)$ is defined by

$$
\begin{aligned}
& W(t) \equiv v(t) u^{\prime}(t)-u(t) v^{\prime}(t) \\
& =v_{m}(t) u_{m}^{\prime}(t)-u_{m}(t) v_{m}^{\prime}(t) \\
& =\frac{1}{2} e^{-t\left(\sqrt{4 k^{2}+n^{2}}+n\right)}\left(\begin{array}{c}
m\left(\sqrt{4 k^{2}+n^{2}}+n\right) U\left(\frac{n m}{2 \sqrt{4 k^{2}+n^{2}}}+\frac{m}{2}+1, m+1, \sqrt{4 k^{2}+n^{2}} t\right) \\
\times L_{\frac{1}{2} m\left(-\frac{n}{\sqrt{4 k^{2}+n^{2}}}-1\right)}\left(t \sqrt{4 k^{2}+n^{2}}\right) \\
-2 \sqrt{4 k^{2}+n^{2}} U\left(\frac{1}{2} m\left(\frac{n}{\sqrt{4 k^{2}+n^{2}}}+1\right), m, \sqrt{4 k^{2}+n^{2}} t\right) \\
\times L_{-\frac{m n}{2 \sqrt{4 k^{2}+n^{2}}}-\frac{m}{2}-1}^{m}\left(t \sqrt{4 k^{2}+n^{2}}\right)
\end{array}\right) \\
& =W(1) e^{n-n t} t^{-m} \text {, }
\end{aligned}
$$

where, obviously, $W(1)$ depends on $n, m$ and $k$.

\section{Some properties of the Green's function}

In this section, we present some properties of the Green's function as theorems which are extremely important in the analysis of (1.1)-(1.2).

Lemma 3.1 Taking into account $A>0, B \geq 0, m>0$ and $n \in \mathbb{R}$, the functions $u(x)$ and $v(x)$, given by (2.7)-(2.8), satisfy the following properties:

- The function $v(x)$ is a positive decreasing function of $x \in[0,1]$ and unbounded at the origin.

- Assuming that $m, n$ and $k$ are such that $W(1)$ is positive, then $u(x)$ is a positive increasing function of $x \in[0,1]$.

- Assuming that $m, n$ and $k$ are such that $W(1)$ is negative, then $u(x)$ is a negative decreasing function of $x \in[0,1]$.

Proof It is straightforward from definitions (2.7)-(2.8) and Wronskian $W(t)$ at $t=1$.

Theorem 3.2 The Green's function $G(x, t)$ is always non-negative, i.e.,

$$
\forall k^{2}, m>0, \forall n \in \mathbb{R}, \forall x, t \in[0,1]: \quad G(x, t) \geq 0 .
$$

Proof Equation (2.16) can be rewritten as follows:

$$
G(x, t)=\frac{e^{n(t-1)} t^{m}}{W(1)} \begin{cases}u(x) v(t), & x \leq t \\ v(x) u(t), & x \geq t\end{cases}
$$

Now, there are two cases.

First, suppose that $m, n$ and $k$ are such that $W(1)$ is positive, then from Lemma 3.1 it follows that $u(x)$ is a positive function of $x \in[0,1]$. On the other hand, $v(x)$ is a positive decreasing function of $x \in[0,1]$ from Lemma 3.1. Therefore, we conclude that $G(x, t) \geq 0$.

Second, suppose that $m, n$ and $k$ are such that $W(1)$ is negative, then from Lemma 3.1 it follows that $u(x)$ is a negative function of $x \in[0,1]$. On the other hands, $v(x)$ is a positive decreasing function of $x \in[0,1]$ from Lemma 3.1. Therefore, we conclude again that $G(x, t) \geq 0$. 
Theorem 3.3 The Green's function $G(x, t)$ is bounded in such a way that

$$
\forall k^{2}, m>0, \forall n \in \mathbb{R}, \forall x, t \in[0,1]: \quad k^{2} \int_{0}^{1} G(x, t) \mathrm{d} t \leq \mu(k)<1 .
$$

Proof Suppose that $\omega(x)$ satisfies differential equation (2.2), then it is easily seen that

$$
\begin{aligned}
\frac{\mathrm{d}}{\mathrm{d} t}\left[t^{m} e^{n t} \omega^{\prime}(t)\right] & =t^{m-1} e^{n t}\left(m \omega^{\prime}(t)+n t \omega^{\prime}(t)+t \omega^{\prime \prime}(t)\right) \\
& =t^{m-1} e^{n t}\left(k^{2} t \omega(t)\right)=k^{2} t^{m} e^{n t} \omega(t),
\end{aligned}
$$

hence

$$
\frac{\mathrm{d}}{\mathrm{d} t}\left[\frac{t^{m} e^{n t} \omega^{\prime}(t)}{W(1) e^{n}}\right]=\frac{k^{2} t^{m} e^{n t} \omega(t)}{W(1) e^{n}}
$$

therefore

$$
\frac{\mathrm{d}}{\mathrm{d} t}\left[\frac{\omega^{\prime}(t)}{W(t)}\right]=\frac{k^{2} \omega(t)}{W(t)} .
$$

Now, with the help of this last equation, we obviously have

$$
k^{2} \int_{0}^{1} G(x, t) \mathrm{d} t=1+\frac{u(x) v^{\prime}(1)}{W(1)} .
$$

Lemma 3.1 confirms that $v^{\prime}(1)<0$ and $\frac{u(x)}{W(1)} \geq 0$, then $\frac{u(x) v^{\prime}(1)}{W(1)}<0$. On the other hand, Theorem 3.2 reveals $\int_{0}^{1} G(x, t) \mathrm{d} t \geq 0$, therefore we conclude that

$$
0 \leq \mu(k)=1+\frac{u(x) v^{\prime}(1)}{W(1)}<1,
$$

and the proof is complete.

\section{The region of existence and uniqueness}

Consider a standard Picard sequence iteration as below which is based on Eq. (2.13)

$$
y_{n+1}(x)=\varphi(x)+\int_{0}^{1} G(x, t)\left[k^{2} y_{n}(t)-f\left(t, y_{n}(t)\right)\right] \mathrm{d} t, \quad n=0,1,2, \ldots
$$

or equivalently in the operator form

$$
y_{n+1}=\varphi(x)+T\left[k^{2} y_{n}-f_{n}\right], \quad n=0,1,2, \ldots,
$$

where $y_{n}=y_{n}(x), f_{n}=f\left(t, y_{n}(x)\right)$, and the operator $T: C[0,1] \rightarrow C[0,1]$ is defined as

$$
T[z(x)]=\int_{0}^{1} G(x, t) z(t) \mathrm{d} t, \quad x \in[0,1] .
$$

Now, we show that (4.1) can converge uniformly in a finite region such as $\mathbf{D}:[0,1] \times$ $\left[y_{L}(x), y_{U}(x)\right]$ so that it presents the unique solution to boundary value problem (1.1)-(1.2) within it. 
Theorem 4.1 Consider boundary value problem (1.1)-(1.2) and suppose the following conditions hold:

(a) $\frac{\partial f}{\partial y}$ is continuous in $\mathbf{D}:[0,1] \times\left[y_{L}(x), y_{U}(x)\right]$ and satisfies $0 \leq \frac{\partial f}{\partial y} \leq N_{D}$ within it.

(b) $y_{0}(x)=\frac{1}{2}\left[y_{L}(x)+y_{U}(x)\right]$ and $y_{L}(x) \leq y_{n}(x) \leq y_{U}(x), n=1,2,3, \ldots$.

(c) The value of $k^{2}$ satisfies $k^{2} \geq \frac{N_{D}}{2}$.

Then the Picard sequence iteration (4.2) converges uniformly to $y(x)$ which is the unique solution of boundary value problem (1.1)-(1.2) in $\mathbf{D}$.

Proof Define

$$
\begin{aligned}
& \Delta y_{n}(x)=y_{n}(x)-y_{n-1}(x), \\
& \Delta f_{n}(x)=f\left(x, y_{n}(x)\right)-f\left(x, y_{n-1}(x)\right),
\end{aligned}
$$

for $n=1,2,3, \ldots$, then by subtracting two successive iterations, we obtain

$$
\Delta y_{n+1}=T\left[k^{2} \Delta y_{n}-\Delta f_{n}\right], \quad n=1,2,3, \ldots
$$

Applying the mean value theorem to $\Delta f_{n}$ results in

$$
\Delta y_{n+1}=T\left[\left(k^{2}-\psi_{n}\right) \Delta y_{n}\right], \quad n=1,2,3, \ldots
$$

where

$$
\psi_{n}(x)=\frac{\partial f}{\partial y}\left[x, y_{n}(x)-\theta(x) \Delta y_{n}(x)\right], \quad 0 \leq \theta(x) \leq 1 .
$$

Remembering $k^{2} \geq \frac{N_{D}}{2}$ and $0 \leq \frac{\partial f}{\partial y} \leq N_{D}$ within $\mathbf{D}$, we conclude $-\frac{N_{D}}{2} \leq k^{2}-\psi_{n} \leq k^{2}$, and then $0 \leq\left|k^{2}-\psi_{n}\right| \leq k^{2}$. Therefore, Eq. (4.7) leads to

$$
\left|\Delta y_{n+1}(x)\right| \leq k^{2} \int_{0}^{1} G(x, t)\left|\Delta y_{n}(t)\right| \mathrm{d} t \leq k^{2}\left\|\Delta y_{n}\right\| \int_{0}^{1} G(x, t) \mathrm{d} t,
$$

where

$$
\|y\|=\max _{0 \leq x \leq 1}|y(x)| .
$$

Now, the application of Theorem 3.3 yields

$$
\left\|\Delta y_{n+1}\right\| \leq \mu(k)\left\|\Delta y_{n}\right\|
$$

In other words,

$$
\left\|\Delta y_{n+1}\right\| \leq[\mu(k)]^{n}\left\|\Delta y_{1}\right\|, \quad \mu(k)<1 .
$$

Now, we have proved that $\left\{y_{n}(x)\right\}$ is a Cauchy sequence with the norm defined by (4.10), then $y_{n}(x)$ converges uniformly to a function $y(x)$ that satisfies integral equation (2.13) or equivalently boundary value problem (1.1)-(1.2). We prove uniqueness of the solution 
by contradiction. Suppose that $y_{1}(x)$ and $y_{2}(x)$ are two solutions to (1.1). Then they easily satisfy (2.13). Now, choosing $k^{2} \geq \frac{N_{D}}{2}$ and using the exact previous analysis that leads to (4.11), we obtain

$$
\left\|y_{1}-y_{2}\right\| \leq \mu(k)\left\|y_{1}-y_{2}\right\|
$$

that is a contradiction because $\mu(k)<1$, and this completes the proof.

\section{Solution procedure and illustrative physiology models}

According to Theorem 4.1, for a given problem (1.1)-(1.2), in order to obtain the solution, a simpler procedure is to obtain relevant $y_{L}(x)$ and $y_{U}(x)$ and then to apply the following Picard iterative sequence:

$$
\begin{aligned}
& y_{0}(x)=\frac{1}{2}\left[y_{L}(x)+y_{U}(x)\right], \\
& y_{n+1}(x)=\varphi(x)+\int_{0}^{1} G(x, t)\left[k^{2} y_{n}(t)-f\left(t, y_{n}(t)\right)\right] \mathrm{d} t, \quad n=0,1,2, \ldots
\end{aligned}
$$

Now, we implement it on two nonlinear singular boundary value problems that arise in real physiology applications.

Example 5.1 Consider the following diffusion problem:

$$
\begin{aligned}
& y^{\prime \prime}(x)+y^{\prime}(x)+\frac{1}{x} y^{\prime}(x)=\exp (y)-1, \\
& y^{\prime}(0)=0, \quad y(1)+y^{\prime}(1)=1 .
\end{aligned}
$$

Assume $y_{L}(x)=0$ and $y_{U}(x)=1$, therefore $y_{0}(x)=\frac{1}{2}$. Also consider $k^{2}=\exp (1)$ because $\frac{\partial f}{\partial y}=\exp (y) \leq \exp (1)$ and continuous in $\mathbf{D}:[0,1] \times\left[y_{L}(x), y_{U}(x)\right]$. Now, it can be verified easily that the assumptions of Theorem 4.1 hold. To this aim, from Eqs. (2.4), (2.7) and (2.11), we obtain

$$
\begin{aligned}
& \varphi(x)=\frac{u(x)}{u(1)+u^{\prime}(1)}, \quad u(x)=e^{\frac{1}{2} x(-\sqrt{4 e+1}-1)} L_{\alpha}^{0}(x \sqrt{4 e+1}), \\
& \alpha=-\frac{(\sqrt{4 e+1}+1)}{2 \sqrt{4 e+1}} .
\end{aligned}
$$

Simple calculations in Mathematica imply that $\varphi(x)$ is an increasing function and $0.33265 \leq \varphi(x) \leq 0.54756$. On the other hand, it holds

$$
\begin{aligned}
\int_{0}^{1} G(x, t)\left[k^{2} y_{n}(t)-f\left(t, y_{n}(t)\right)\right] \mathrm{d} t & =\int_{0}^{1} G(x, t)\left[k^{2} y_{n}(t)+1-\exp \left(y_{n}(t)\right)\right] \mathrm{d} t \\
& =\int_{0}^{1} k^{2} G(x, t)\left[y_{n}(t)+\frac{1-\exp \left(y_{n}(t)\right)}{e}\right] \mathrm{d} t .
\end{aligned}
$$

If $0 \leq y_{n}(x) \leq 1$, then $0 \leq y_{n}(t)+\frac{1-\exp \left(y_{n}(t)\right)}{e} \leq 0.367880$. Then from Theorem 3.3 we have

$$
0 \leq \int_{0}^{1} G(x, t)\left[k^{2} y_{n}(t)-f\left(t, y_{n}(t)\right)\right] \mathrm{d} t \leq 0.367880,
$$




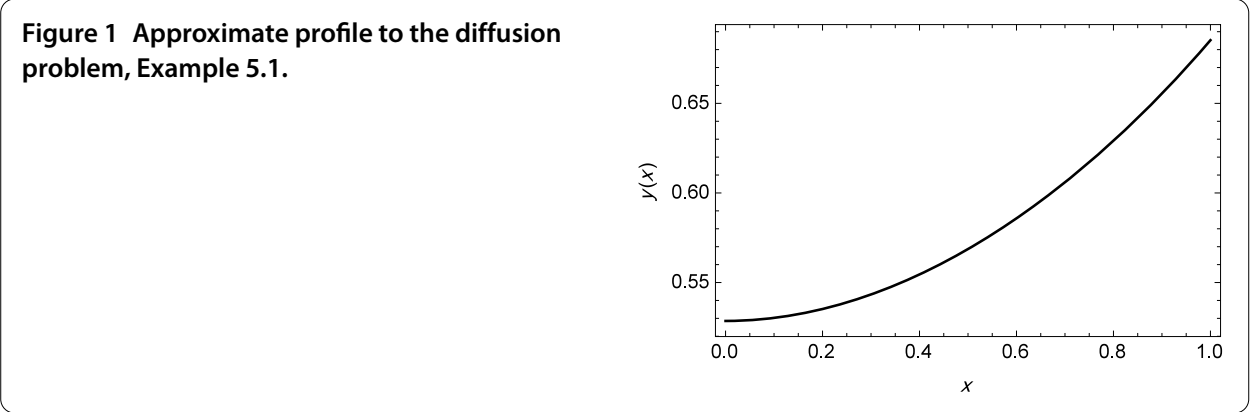

Figure 2 Approximate solution of the diffusion problem, Example 5.2.

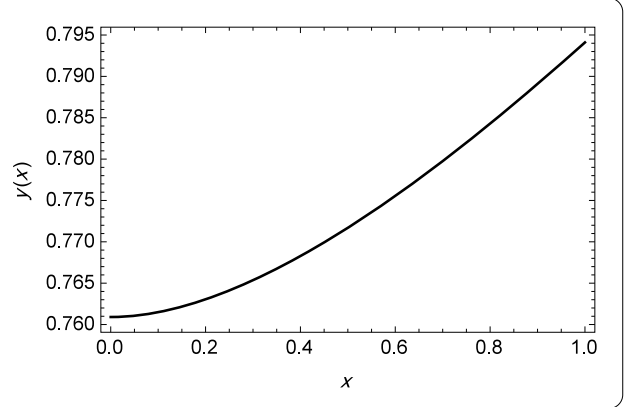

therefore we conclude $0 \leq y_{n+1}(x) \leq 1$ from (5.1). The approximate solution is given in Figure 1.

Example 5.2 Consider the following problem:

$$
\begin{aligned}
& y^{\prime \prime}(x)+10 y^{\prime}(x)+\frac{5}{x} y^{\prime}(x)=y, \\
& y^{\prime}(0)=0, \quad y(1)+4 y^{\prime}(1)=1 .
\end{aligned}
$$

Suppose again $y_{L}(x)=0$ and $y_{U}(x)=1$, thus $y_{0}(x)=\frac{1}{2}$. Moreover, consider $k^{2}=1$ because $\frac{\partial f}{\partial y}=1$ and continuous in $\mathbf{D}:[0,1] \times\left[y_{L}(x), y_{U}(x)\right]$. Now, indeed all the assumptions of Theorem 4.1 hold because

$$
\begin{aligned}
& \varphi(x)=\frac{u(x)}{u(1)+4 u^{\prime}(1)}, \quad u(x)=e^{\frac{1}{2} x(-\sqrt{104}-10)} L_{\alpha}^{4}(x \sqrt{104}), \\
& \alpha=-\frac{5(\sqrt{104}+10)}{2 \sqrt{104}},
\end{aligned}
$$

and then $0.76091 \leq \varphi(x) \leq 0.794105$ while it is increasing in $[0,1]$. Moreover, it can be easily seen that

$$
\int_{0}^{1} G(x, t)\left[k^{2} y_{n}(t)-f\left(t, y_{n}(t)\right)\right] \mathrm{d} t \equiv 0 ;
$$

therefore, obviously, $0 \leq y_{n+1}(x) \leq 1$ from (5.1). The analytical approximate solution to this problem is shown in Figure 2. 
Conflict of interest

The authors declare that there is no conflict of interest regarding the publication of this paper.

\section{Competing interests}

The authors declare that they have no competing interests.

\section{Authors' contributions}

All authors have made the same contribution and finalized the current version of this manuscript.

Funding

The current work is partially supported by Islamic Azad University and Imam Khomeini International University.

\section{Author details}

'Department of Mathematics, Islamic Azad University, Karaj Branch, Karaj, Iran. ${ }^{2}$ Department of Mathematics, Imam Khomeini International University, Qazvin, 34149-16818, Iran.

\section{Acknowledgements}

The authors are grateful to anonymous reviewers for carefully reading this paper and for their comments and suggestions which have improved the paper.

\section{Publisher's Note}

Springer Nature remains neutral with regard to jurisdictional claims in published maps and institutional affiliations.

Received: 1 September 2016 Accepted: 19 April 2017 Published online: 27 April 2017

\section{References}

1. Garner, J, Shivaji, R: Diffusion problems with a mixed nonlinear boundary condition. J. Math. Anal. Appl. 148(2), 422-430 (1990)

2. Adam, JA: A simplified mathematical model of tumor growth. Math. Biosci. 81(2), 229-244 (1986)

3. Adam, JA: A mathematical model of tumor growth. II. Effects of geometry and spatial nonuniformity on stability. Math. Biosci. 86(2), 183-211 (1987)

4. Adam, JA, Maggelakis, S: Mathematical models of tumor growth. IV. Effects of a necrotic core. Math. Biosci. 97(1), 121-136 (1989)

5. Burton, AC: Rate of growth of solid tumours as a problem of diffusion. Growth 30(2), 157-176 (1966)

6. Greenspan, H: Models for the growth of a solid tumor by diffusion. Stud. Appl. Math. 51(4), 317-340 (1972)

7. Asaithambi, N, Garner, J: Pointwise solution bounds for a class of singular diffusion problems in physiology. Appl. Math. Comput. 30(3), 215-222 (1989)

8. McElwain, D: A re-examination of oxygen diffusion in a spherical cell with Michaelis-Menten oxygen uptake kinetics. J. Theor. Biol. 71(2), 255-263 (1978)

9. Flesch, U: The distribution of heat sources in the human head: a theoretical consideration. J. Theor. Biol. 54(2), 285-287 (1975)

10. Gray, B: The distribution of heat sources in the human head - theoretical considerations. J. Theor. Biol. 82(3), 473-476 (1980)

11. Duggan, R, Goodman, A: Pointwise bounds for a nonlinear heat conduction model of the human head. Bull. Math. Biol. 48(2), 229-236 (1986)

12. Gatica, J, Oliker, V, Waltman, P: Singular nonlinear boundary value problems for second-order ordinary differential equations. J. Differ. Equ. 79(1), 62-78 (1989)

13. Fink, A, Gatica, JA, Hernandez, GE, Waltman, P: Approximation of solutions of singular second order boundary value problems. SIAM J. Math. Anal. 22(2), 440-462 (1991)

14. Baxley, JV: Some singular nonlinear boundary value problems. SIAM J. Math. Anal. 22(2), 463-479 (1991)

15. Baxley, JV, Gersdorff, GS: Singular reaction-diffusion boundary value problems. J. Differ. Equ. 115(2), $441-457$ (1995)

16. Wang, $\mathrm{H}, \mathrm{Li}$, Y: Existence and uniqueness of solutions to two point boundary value problems for ordinary differential equations. Z. Angew. Math. Phys. 47(3), 373-384 (1996)

17. Tineo, A: On a class of singular boundary value problems which contains the boundary conditions. J. Differ. Equ. 113, 1-16 (1994)

18. Wang, J: On positive solutions of singular nonlinear two-point boundary value problems. J. Differ. Equ. 107(1), 163-174 (1994)

19. Ebaid, A: A new analytical and numerical treatment for singular two-point boundary value problems via the Adomian decomposition method. J. Comput. Appl. Math. 235(8), 1914-1924 (2011)

20. Agarwal, RP, O'Regan, D: Nonlinear superlinear singular and nonsingular second order boundary value problems. J. Differ. Equ. 143(1), 60-95 (1998)

21. Jia, R, Shao, J: Existence and uniqueness of periodic solutions of second-order nonlinear differential equations. J. Inequal. Appl. 2013, 115 (2013)

22. Zhou, J: The existence and uniqueness of the solution for nonlinear elliptic equations in Hilbert spaces. J. Inequal. Appl. 2015, $250(2015)$

23. Agarwal, RP, O'Regan, D: An upper and lower solution approach for singular boundary value problems with sign changing non-linearities. Math. Methods Appl. Sci. 25(6)، 491-506 (2002)

24. Kannan, $\mathrm{R}, \mathrm{O}$ 'Regan, $\mathrm{D}$ : Singular and nonsingular boundary value problems with sign changing nonlinearities. J. Inequal. Appl. 5, 621-637 (2000)

25. Lü, H, Bai, Z: Positive radial solutions of a singular elliptic equation with sign changing nonlinearities. Appl. Math. Lett. 19(6), 555-567 (2006)

26. Ford, WF, Pennline, JA: Singular non-linear two-point boundary value problems: existence and uniqueness. Nonlinear Anal. 71(3), 1059-1072 (2009) 